\title{
Composition atmosphérique et bilan radiatif
}

Jean Poitou (jean.poitou@wanadoo.fr)

Ancien adjoint au directeur du Laboratoire des Sciences du Climat et de I'Environnement

Le climat de la Terre résulte de l'équilibre entre l'énergie solaire qu'elle absorbe et l'énergie qui repart vers l'espace sous forme de rayonnement infrarouge ; près de 90\% de l'énergie sortante est émise par les couches supérieures de I'atmosphère, à une altitude qui croît avec la concentration des gaz à effet de serre (GES).

La température de cette zone émettrice diminue avec l'altitude : I'accumulation de GES se traduit donc par une émission moindre d'infrarouges.

Le forçage radiatif - nom donné au déséquilibre ainsi produit réchauffe toute la colonne atmosphérique. Les impacts complexes des aérosols provoquent d'autres forçages indirects, dont le bilan est un effet refroidissant. Les forçages d'origine anthropique dominent ceux d'origine naturelle,

et sont en augmentation

ininterrompue du fait de

I'accumulation des GES dans

l'atmosphère.
Au cours du dernier siècle, la température moyenne de la Terre a augmenté de $0,7^{\circ} \mathrm{C}$. Cette augmentation peut paraître minime ; mais, d'une part, elle été très rapide par rapport aux évolutions qu'a connu le climat dans les époques passées ; d'autre part, elle semble bien n'être que le prélude à un réchauffement nettement plus conséquent susceptible d'affecter la Terre pendant des millénaires. Le but de cet article est de comprendre comment l'accroissement de la concentration atmosphérique de gaz qui y sont présents à l'état de traces, peut perturber le bilan radiatif et par là le climat de la Terre, alors que les rayonnements infrarouges concernés sont déjà presque complètement absorbés par l'atmosphère. En d'autres termes, comment l'adjonction par l'homme de gaz à effet de serre augmente significativement et durablement l'effet de serre.

La Terre reçoit toute son énergie du Soleil ${ }^{(a)}$. Le Soleil a une température de surface de $5780 \mathrm{~K}$. Il émet un rayonnement électromagnétique, essentiellement de corps noir, sauf aux courtes longueurs d'onde où d'autres processus d'émission entrent en jeu. Au niveau de la Terre, le flux lumineux solaire est de $1360 \mathrm{~W} . \mathrm{m}^{-2}$ pour une surface perpendiculaire aux rayons du Soleil. Répartie à la surface du globe, en moyennant sur les cycles diurnes et saisonniers, cela donne un flux moyen de $340 \mathrm{~W} . \mathrm{m}^{-2}$.

Approximativement 30\% de ce rayonnement incident est rediffusé vers l'espace, par l'atmosphère (diffusion par les molécules, par les nuages, par les aérosols) et par la surface du globe. Le reste, soit 70\% du flux incident, est absorbé dans l'atmosphère ou par la surface du globe et converti en chaleur. La Terre (globe terrestre plus atmosphère) évacue la chaleur sous forme de rayonnement électromagnétique, le seul processus possible de transfert d'énergie vers l'espace, par rayonnement de corps noir. Les conditions climatiques s'ajustent pour réaliser un bilan énergétique, énergie entrante moins énergie sortante, nul en moyenne. Avec un flux de rayonnement incident de 340 W.m. ${ }^{-2}$ et un albédo ${ }^{(b)}$ de $30 \%$, la loi de Stefan-Boltzmann ${ }^{(c)}$ donne $\mathrm{T}=255 \mathrm{~K}$, soit $-18^{\circ} \mathrm{C}$, une température nettement inférieure à la température moyenne de surface du globe, qui est actuellement de $288 \mathrm{~K}$, soit $+15^{\circ} \mathrm{C}$. Cette différence de température résulte de l'effet de serre ${ }^{(\mathrm{d})}$.

Composition de l'air. Les pourcentages des gaz autres que la vapeur d'eau sont donnés pour l'air sec. En violet, les gaz qui absorbent des infrarouges. tableau 1

\begin{tabular}{|c|c|c|}
\hline Gaz & Formule chimique & Abondance (volume) en \% \\
\hline Azote & $\mathrm{N}_{2}$ & 78,08 \\
\hline Oxygène & $\mathrm{O}_{2}$ & 20,95 \\
\hline Vapeur d'eau & $\mathrm{H}_{2} \mathrm{O}$ & 0 à 4 (dépend de la température) \\
\hline Argon & $\mathrm{Ar}$ & 0,93 \\
\hline Dioxyde de carbone & $\mathrm{CO}_{2}$ & 0,039 \\
\hline Néon & $\mathrm{Ne}$ & 0,0018 \\
\hline Hélium & $\mathrm{He}$ & 0,0005 \\
\hline Méthane & $\mathrm{CH}_{4}$ & 0,00017 \\
\hline Krypton & $\mathrm{Kr}$ & 0,0001 \\
\hline Xénon & $\mathrm{Xe}$ & 0,00008 \\
\hline Hydrogène & $\mathrm{H}_{2}$ & 0,00005 \\
\hline Oxyde nitreux & $\mathrm{N}_{2} \mathrm{O}$ & 0,00003 \\
\hline Ozone & $\mathrm{O}_{3}$ & 0,000004 \\
\hline
\end{tabular}



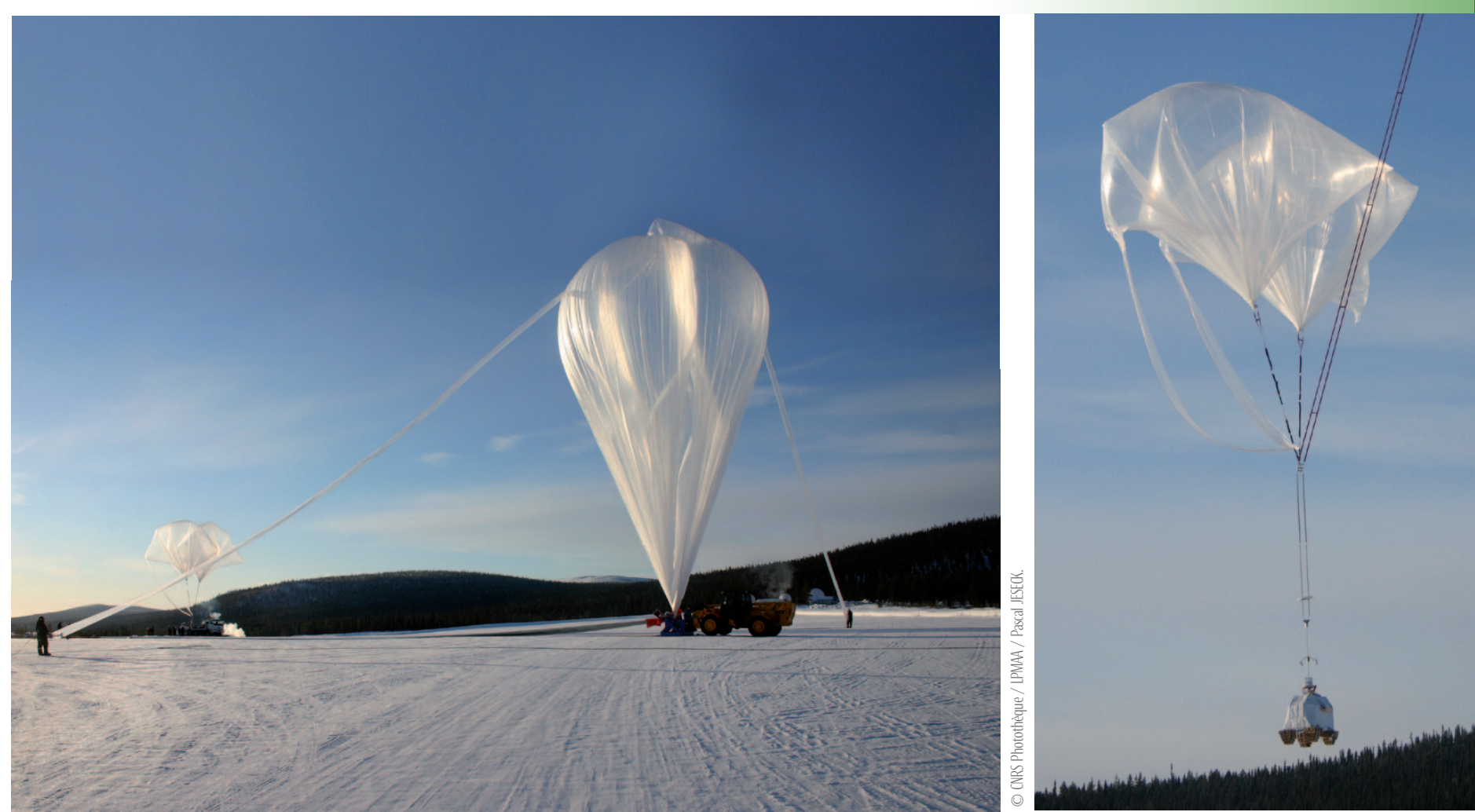

L'expérience SWIR-ballon du LPMAA (UMR 7092 CNRS-UP6) sur l'aire de lancement à Kiruna en Suède. L'instrument scientifique principal est un spectromètre infrarouge à transformée de Fourier, pour mesurer le rayonnement solaire diffusé par les molécules de l'atmosphère. Le but de cette expérience est de déterminer les concentrations de deux gaz à effet de serre, le dioxyde de carbone $\left(\mathrm{CO}_{2}\right)$ et le méthane $\left(\mathrm{CH}_{4}\right)$.

\section{L'effet de serre atmosphérique $e^{(e)}$}

Les informations nécessaires pour comprendre le fonctionnement de l'effet de serre sont présentées dans le tableau 1 et la figure 1. L'atmosphère est composée essentiellement d'azote et d'oxygène, mais aussi d'un certain nombre de gaz à l'état de traces (tableau 1) qui présentent des raies d'absorption, essentiellement des modes de vibration, dans la gamme de longueurs d'onde de l'infrarouge thermique, c'est-àdire au-delà de 2,5 $\mu \mathrm{m}$. C'est le cas des molécules dissymétriques et de celles constituées de trois atomes ou plus.

Du point de vue spectral, la lumière venant du Soleil s'étend de l'ultraviolet au proche infrarouge. Une part appréciable des courtes longueurs d'onde du spectre solaire est absorbée par l'ozone dans la stratosphère ou affectée par la diffusion de Rayleigh, diffusion de la lumière sur les molécules du gaz, dont l'amplitude varie comme $\lambda^{-4}$ ( $\lambda$ est la longueur d'onde). En l'absence de nuages et d'aérosols, l'atmosphère est transparente pour la plus grande partie du reste du spectre solaire dans le visible et le proche infrarouge, comme le montre la figure 1.
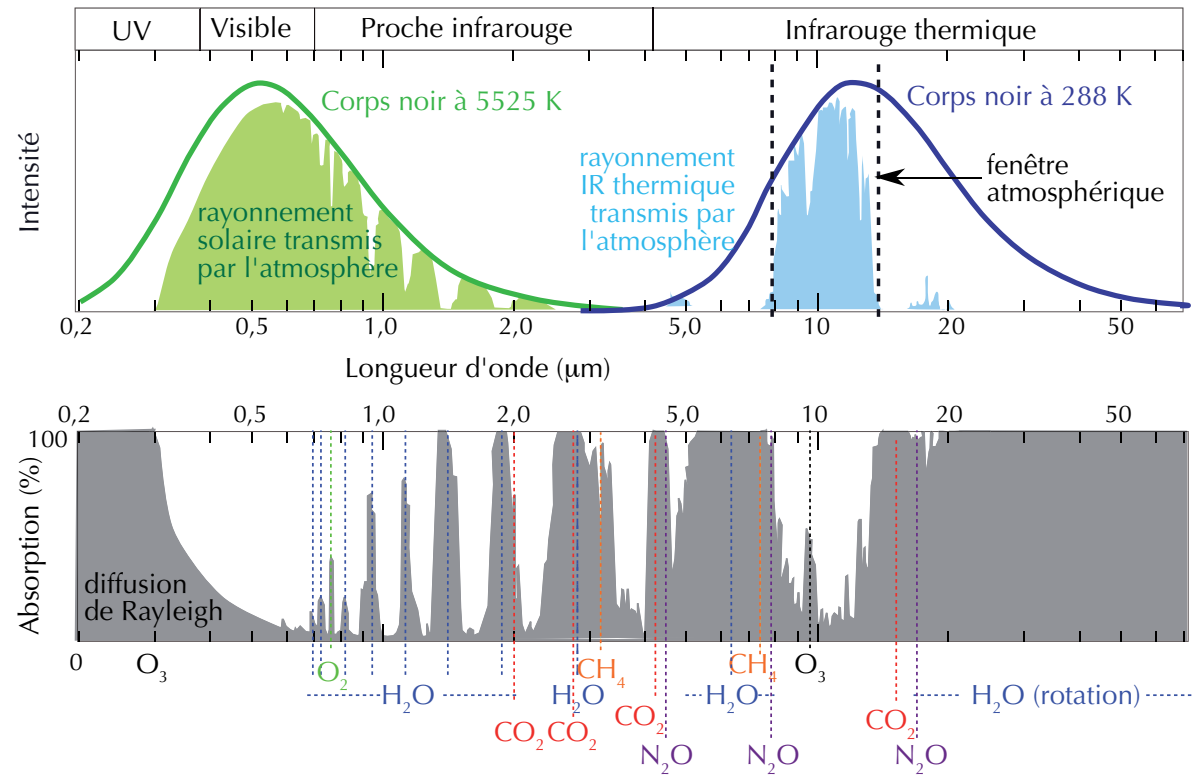

1. Spectres d'émission et d'absorption par l'atmosphère du rayonnement solaire et du rayonnement émis par le sol terrestre, en l'absence de nuages et d'aérosols. En haut : en vert, spectre d'émission du corps noir proche du spectre d'émission du Soleil, et la fraction atteignant la surface terrestre après traversée de l'atmosphère ; en bleu, spectre d'émission à 288 K, température moyenne de la surface terrestre, et la fraction qui ressort dans l'espace; noter la « fenêtre atmosphérique " autour de 10 um.

En bas : absorption par la colonne atmosphérique du rayonnement émis au niveau du sol. Les domaines de contributions des principaux gaz naturellement présents dans l'atmosphère sont indiqués sous la figure pour le domaine de longueurs d'ondes du rayonnement solaire et du rayonnement thermique terrestre. L'atmosphère piège aussi une partie du rayonnement aux courtes longueurs d’onde à cause de la diffusion de la lumière par les molécules (diffusion de Rayleigh). 


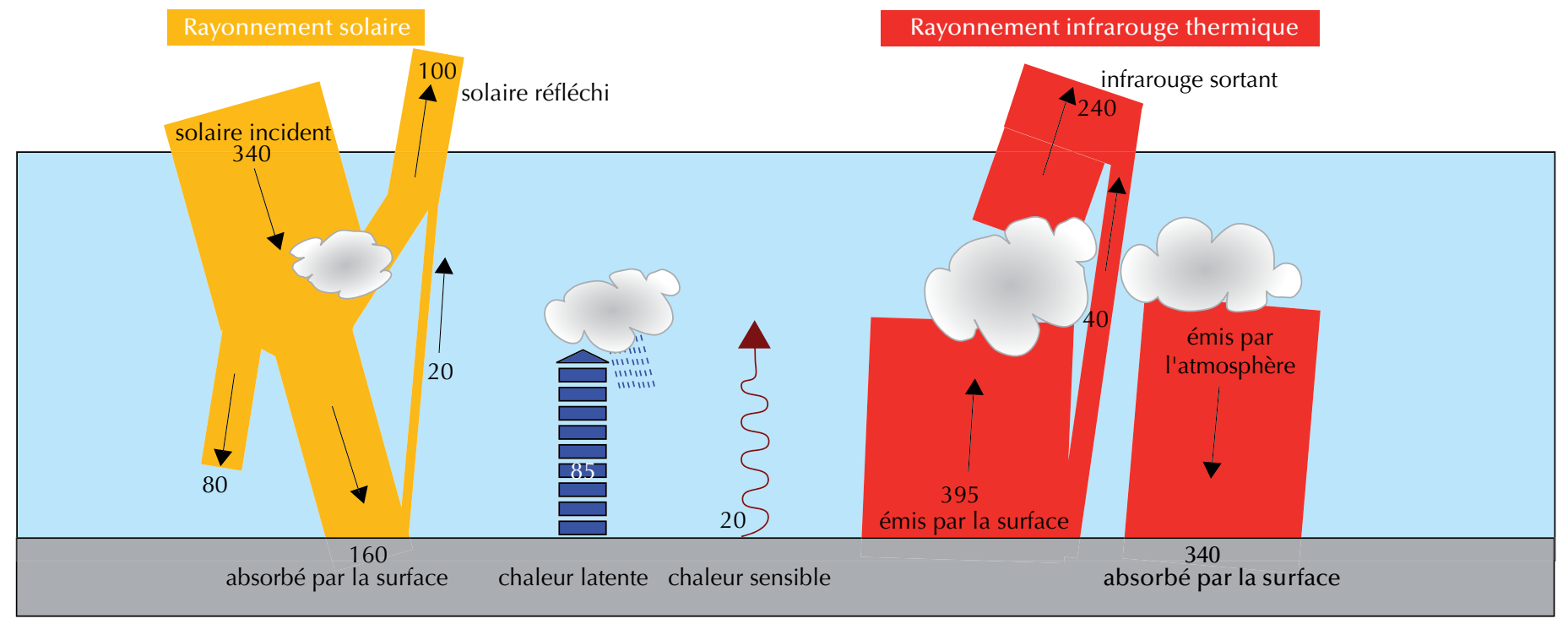

2. Représentation schématique des échanges d'énergie entre la Terre, l'atmosphère et l'espace. L'épaisseur des bandes rouges et jaunes est représentative des flux en jeu, indiqués par les flèches et exprimés en $\mathrm{W} \cdot \mathrm{m}^{-2}$. Le flux de $40 \mathrm{~W} / \mathrm{m}^{2}$ (bande rouge) qui va directement vers l'espace correspond à la «fenêtre atmosphérique ".

$>>$

Pour une grande part des longueurs d'onde du rayonnement thermique émis par la Terre (au-delà de 2,5 $\mu \mathrm{m}$ ), l'atmosphère est opaque (l'absorption est dite "saturée »), sauf essentiellement dans un domaine de longueurs d'onde restreint, entre 8 et $14 \mu \mathrm{m}$, la "fenêtre atmosphérique ", bien visible sur la figure 1, qui est utilisée pour les observations spatiales de la Terre, par exemple par les satellites météorologiques.

Quels sont les processus en jeu ? La figure 2 montre de façon schématique l'ensemble des échanges de chaleur entre l'espace, la surface terrestre et l'atmosphère. Les échanges de chaleur entre la Terre et l'atmosphère ne se limitent pas aux échanges radiatifs. Comme le montre la figure 2, la surface du globe transmet de la chaleur à l'atmosphère par rayonnement, mais aussi sous forme de chaleur sensible (la chaleur que nous ressentons, transmise par conduction ou transportée par convection), et surtout de chaleur latente, émise principalement au-dessus des océans.

L'atmosphère étant absorbante pour le rayonnement, elle est aussi émettrice, conformément à la loi de Kirchhoff qui stipule que tout corps qui est absorbant pour un rayonnement électromagnétique est également émetteur de ce même rayonnement. L'atmosphère émet donc du rayonnement infrarouge et elle le fait dans toutes les directions. La moitié descend vers la surface du globe et sera absorbée par l'atmosphère et la surface ; l'autre moitié se dirige vers l'espace. Tant que la quantité de gaz à effet de serre située au-dessus du point d'émission est suffisamment importante, tout le rayonnement ascendant émis au sein de l'atmosphère est à son tour absorbé. Ne sortira de l'atmosphère que le rayonnement émis à une altitude telle qu'il ne rencontre plus une quantité de gaz à effet de serre suffisante pour l'absorber complètement. Pour une concentration donnée de gaz à effet de serre, on peut définir une altitude effective d'émission, qui est celle à laquelle sera émis, en moyenne, le rayonnement qui partira vers l'espace. Cette altitude effective va croître avec la concentration en gaz absorbants. Il faut garder en tête que, alors que les gaz comme le $\mathrm{CO}_{2}$ ou le $\mathrm{CH}_{4}$ sont bien mélangés, la vapeur d'eau se trouve majoritairement à basse altitude. Ce sont donc essentiellement ces gaz bien mélangés qui vont déterminer l'altitude effective d'émission.

L'intensité du rayonnement émis par l'atmosphère est conditionnée par le profil vertical de température, décroissante avec l'altitude. Le flux d'énergie émis variant, selon la loi de Stefan-Boltzmann, comme la puissance 4 de la température absolue, l'intensité du rayonnement émis décroît sensiblement avec l'altitude d'émission. Ce qui sort des couches supérieures de l'atmosphère vers l'espace ne représente que $60 \%$ de ce que la surface émet sous forme de rayonnement infrarouge et moins de $50 \%$ de l'énergie totale qui quitte la surface. Le bilan radiatif à la surface est fermé grâce à la fraction descendante du rayonnement infrarouge émis par l'atmosphère (fig. 2).

Que se passe-t-il quand la concentration de gaz absorbant varie (fig. 3) ? Prenons le cas du $\mathrm{CO}_{2}$ qui est bien mélangé dans l'atmosphère ; l'augmentation de la concentration de $\mathrm{CO}_{2}$ est la même sur toute la hauteur de la troposphère. L'altitude effective varie de façon à ce que la quantité intégrée de $\mathrm{CO}_{2}$ au-dessus de cette altitude reste constante. En l'absence de réaction du climat, on aurait donc émission à une température plus basse et donc un flux d'énergie sortante moindre ; le déséquilibre ainsi créé est appelé forçage radiatif. Le rétablissement de l'équilibre radiatif implique que le flux d'énergie émis reste le même, ce qui veut dire que la température de la nouvelle zone émettrice, à plus haute altitude, doit être la même que celle de l'ancienne zone émettrice. L'adjonction de $\mathrm{CO}_{2}$ n'affecte pas les conditions de stabilité verticale de l'atmosphère, le gradient thermique est conservé. Il faut donc que la température de toute la colonne atmosphérique augmente avec la concentration des gaz à effet de serre, y compris au voisinage de la surface. C'est une conséquence du caractère saturé de l'absorption ; quand la concentration est assez basse pour que l'absorption soit loin d'être saturée, l'adjonction de gaz absorbant fait, conformément à la loi de Kirchhoff, que la température décroît, ce qui est le cas dans la stratosphère.

Le calcul quantitatif de l'effet de serre est évidemment plus complexe que cette explication avec les mains. Il fait appel aux lois de la thermodynamique et aux interactions des molécules avec le rayonnement. Un des résultats de ce calcul est le pouvoir de réchauffement global des différents gaz (voir encadré 1). 


\section{Le forçage radiatif}

Dans une situation d'équilibre du climat, le flux d'énergie entrante, provenant du Soleil, est égal au flux d'énergie sortante, composée de la fraction d'énergie entrante qui est rétrodiffusée et du rayonnement infrarouge émis par la Terre, surface + atmosphère. Une perturbation qui modifie l'un des deux flux : variation de la luminance solaire, altération de la transparence de l'atmosphère, modification de l'albédo terrestre... va rompre cet équilibre comme on vient de le voir dans le cas d'ajout de gaz à effet de serre. On désigne sous le nom de forçage radiatif le déséquilibre sur le bilan énergétique : [flux entrant] - [flux sortant], provoqué par une modification de l'environnement avant que le climat ait réagi pour rétablir l'équilibre.

Depuis la révolution industrielle, l'homme injecte dans l'atmosphère des quantités massives de dioxyde de carbone (34 milliards de tonnes en 2011) et d'autres gaz à effet de serre. L'évolution du forçage lié aux principaux gaz injectés par l'homme dans l'atmosphère est portée sur la figure 4 . La tendance quasi linéaire pour le $\mathrm{CO}_{2}$ en coordonnées logarithmiques indique en fait une augmentation exponentielle du forçage résultant d'une augmentation quasi continue des émissions, comme le montre l'histogramme du bas de la figure 4. La situation n'est pas la même pour tous les gaz à effet de serre émis par l'homme. Le méthane a peu augmenté dans la dernière décennie, mais semble repartir à la hausse. Les émissions de gaz industriels comme les chlorofluorocarbures ont fortement progressé à partir des années 1940 ; le protocole de Montréal destiné à protéger la couche d'ozone a donné un sérieux coup de frein à leur augmentation, mais les gaz destinés à les remplacer sont aussi de puissants gaz à effet de serre ; ces divers gaz ont des bandes d'absorption dans la «fenêtre atmosphérique ".

L'ensemble des forçages par rapport à la situation en 1750 est porté sur la figure 5 . On distingue le forçage radiatif tel que décrit ci-dessus, et le forçage ajusté, qui, prenant en compte l'évolution des agents forçants considérés et leur impact sur la physique de l'atmosphère, donnent une meilleure évaluation de l'effet radiatif induit pas ces agents (voir encadré 2, p. 33). Cette figure montre l'importance prédominante des gaz à effet de serre par rapport à tous les autres forçages. Il faut remarquer l'effet refroidissant des aérosols (fines particules

\section{Pouvoir de réchauffement global (PRG)} encadré 1

Pour comparer l'action des différents gaz à effet de serre, on a introduit le concept de pouvoir de réchauffement global. Partant de l'atmosphère actuelle prise comme référence, on y ajoute différents gaz à effet de serre. L'adjonction aujourd'hui d'une masse $\boldsymbol{M}$ de $\mathrm{CO}_{2}$ va conduire dans $n$ années à un réchauffement cumulé $\boldsymbol{R}$. Si, au lieu de $\mathrm{CO}_{2}$, on ajoute la même masse d'un autre gaz $G$, le réchauffement obtenu sera $R^{\prime}$. Le rapport $R^{\prime} / R$ est le PRG du gaz $G$ à l'échéance de $n$ années. Dans les rapports du GIEC, $\boldsymbol{n}$ est égal à 100 ans.

Le temps de résidence d'un gaz injecté dans I'atmosphère conditionne fortement son PRG. Ainsi, la vapeur d'eau qui ne séjourne qu'une quinzaine de jours dans l'atmosphère avant d'en être éliminée par précipitation a un PRG négligeable. Le méthane, dont la durée de vie avant oxydation par les radicaux $\mathrm{OH}$ de l'atmosphère est d'une dizaine d'années, aura un PRG d'autant plus important que l'échéance considérée sera plus proche, le $\mathrm{CO}_{2}$ produit ayant un pouvoir instantané beaucoup plus faible que le méthane.

Le tableau donne les temps de résidence et les PRG de divers gaz à effet de serre produits par l'homme. Ces PRG sont calculés par les codes de transfert radiatif dans l'atmosphère.

\begin{tabular}{|c|c|c|c|c|}
\hline Gaz & $\begin{array}{c}\text { Temps de } \\
\text { résidence } \\
\text { (ans) }\end{array}$ & $\begin{array}{c}\text { PRG } \\
\text { à } \mathbf{2 0} \text { ans }\end{array}$ & $\begin{array}{c}\text { PRG } \\
\text { à } \mathbf{1 0 0} \text { ans }\end{array}$ & $\begin{array}{c}\text { PRG } \\
\text { à } \mathbf{5 0 0} \text { ans }\end{array}$ \\
\hline Dioxyde de carbone $: \mathrm{CO}_{2}$ & $>1$ siècle & 1 & 1 & 1 \\
\hline Méthane $: \mathrm{CH}_{4}$ & 12 & 72 & 25 & 7,6 \\
\hline Oxyde nitreux $: \mathrm{N}_{2} \mathrm{O}$ & 114 & 289 & 298 & 153 \\
\hline CFC-11: $\mathrm{CCl}_{3} \mathrm{~F}$ & 45 & 6730 & 4750 & 1620 \\
\hline CFC-115: $\mathrm{CClF}_{2} \mathrm{CF}_{3}$ & 1700 & 5310 & 7370 & 9990 \\
\hline HCFC-22: $\mathrm{CHClF}_{2}$ & 12 & 5160 & 1810 & 549 \\
\hline HFC-23: $\mathrm{CHF}_{3}$ & 270 & 12000 & 14800 & 12200 \\
\hline Hexafluorure de soufre $: \mathrm{SF}_{6}$ & 3200 & 16300 & 22800 & 32600 \\
\hline Trifluorure d'azote $: \mathrm{NF}_{3}$ & 740 & 12300 & 17200 & 20700 \\
\hline PFC-14: $\mathrm{CF}_{4}$ & 50000 & 5210 & 7390 & 11200 \\
\hline
\end{tabular}

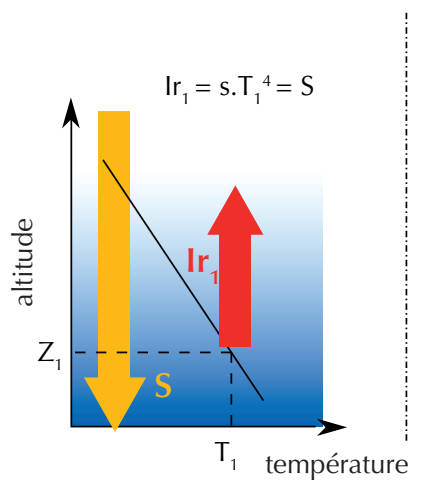

concentration de gaz à effet de serre accrue

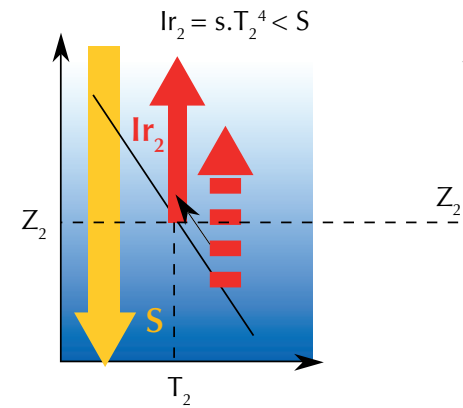

$\mathrm{Ir}_{3}=\mathrm{s} \cdot \mathrm{T}_{1}^{4}=\mathrm{S}=\mathrm{Ir} \mathrm{r}_{1}$

$\mathrm{S}=$ flux solaire net absorbé $=$ flux entrant - flux réfléch

Ir = flux infrarouge thermique sortant $\quad \mathrm{Ir}_{2}-\mathrm{S}=$ forçage radiatif

3. Effet sur le bilan radiatif et le climat d'un accroissement de la concentration atmosphérique de Gaz à Effet de Serre (GES). L'intensité du bleu symbolise la concentration volumique du GES ; l'épaisseur des flèches symbolise le flux d'énergie. La ligne continue noire représente la variation de température de l'atmosphère avec l'altitude. Partons d'un climat à l'équilibre (à gauche). Le rayonnement qui sort de l'atmosphère est émis à l'altitude $Z_{1}$ à une température $T_{1}$. Ajoutons du GES (figure du milieu). La quantité de GES au-dessus de $Z_{1}$ est trop grande pour que le rayonnement puisse sortir. Le rayonnement sortant est émis à $Z_{2}$. Du fait du gradient thermique vertical de l'atmosphère, la température $T_{2}$ est plus faible que $T_{1}$ et par conséquent le flux d’énergie émis est plus faible. II y a déséquilibre entre le rayonnement entrant et le rayonnement sortant. On a donc accumulation de chaleur et, du fait du gradient thermique qui n'est pas affecté par la présence de GES, toute la colonne atmosphérique se réchauffe pour rétablir l'équilibre (à droite). 


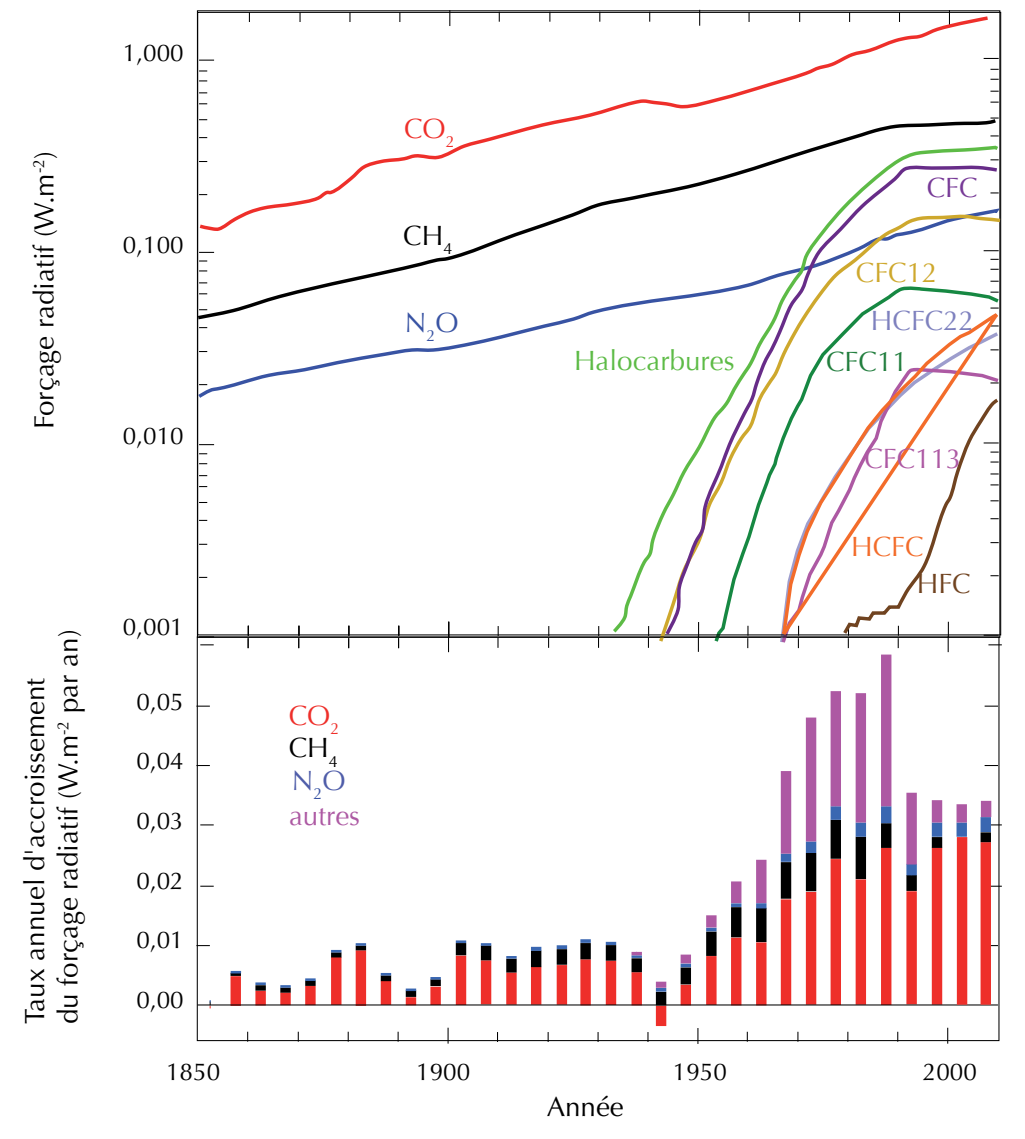

4. Évolution depuis $\mathbf{1 8 5 0}$ du forçage induit par les différents gaz à effet de serre produits par l'homme. En haut : forçage en coordonnées logarithmiques. En bas : taux annuel d'accroissement du forçage.

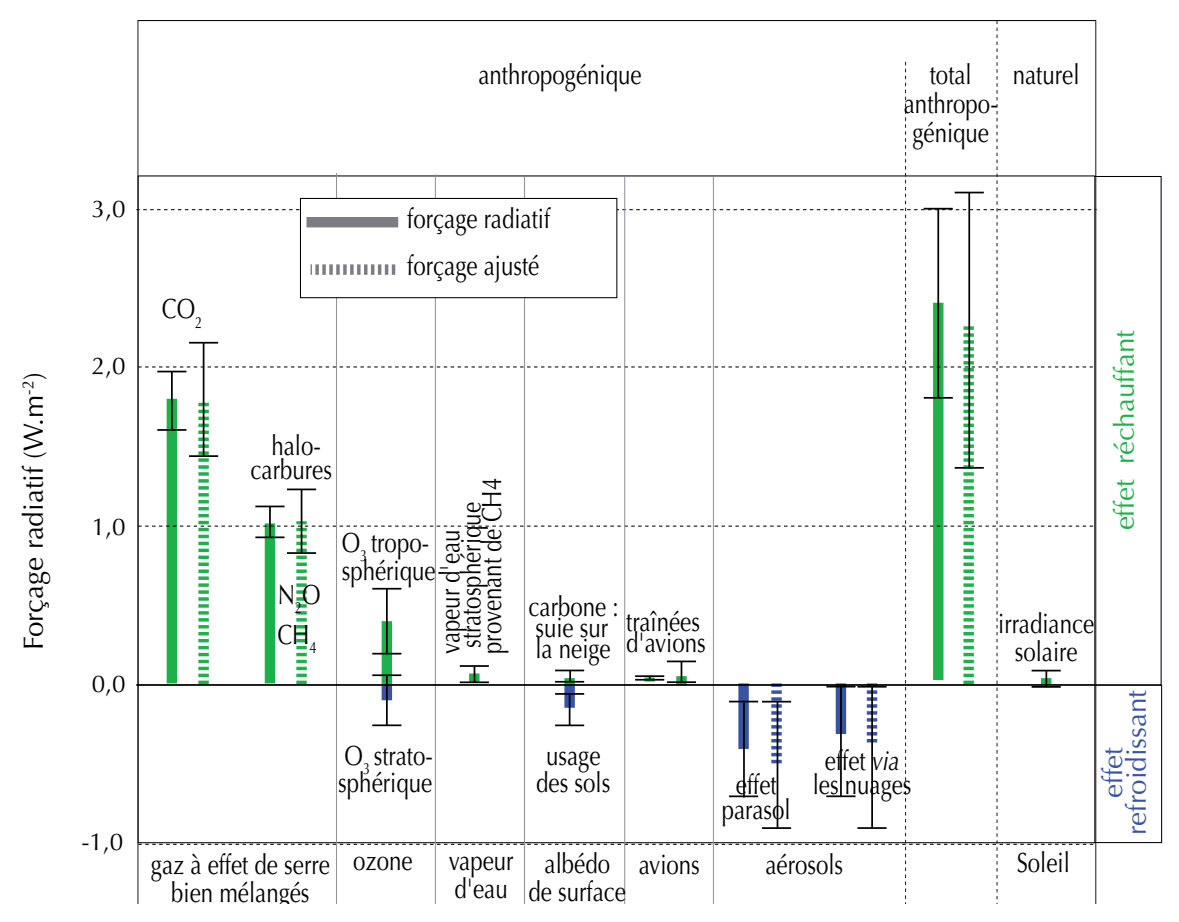

5. Les diverses composantes du forçage radiatif (barres pleines), et du forçage ajusté (voir encadré 2) en barres tiretées, pour la situation en 2011 par rapport à la situation en 1750 prise comme référence. $\gg>$

en suspension dans l'atmosphère), particulièrement marqué même s'il est entaché d'incertitudes appréciables, et grâce auquel la température moyenne de la Terre n'a encore augmenté que modestement $\left(0,7^{\circ} \mathrm{C}\right.$ en un siècle) ; c'est en quelque sorte un effet bénéfique de la pollution, par ailleurs délétère pour la santé. On notera aussi sur la figure 5 la faiblesse du forçage lié à l'irradiance solaire dont les variations d'un cycle solaire de 11 ans à l'autre sont minimes $-0,1 \mathrm{~W} \cdot \mathrm{m}^{-2}$ hors de l'atmosphère, $0,0175 \mathrm{~W} \cdot \mathrm{m}^{-2}$ au sol - et très faibles par rapport au forçage anthropique ; les variations de cette irradiance au cours du cycle solaire, dix fois plus élevées, sont sans effet sur la tendance à long terme de la température moyenne globale.

Comment a évolué le forçage depuis 1750 ? C'est ce qui est montré sur la figure 6 pour chacune de ses composantes. Se superposant aux forçages produits par l'action de l'homme, on note une contribution minime du Soleil. Mais un des effets les plus frappants est le fort refroidissement produit par les éruptions volcaniques majeures : les particules injectées dans la stratosphère forment un écran aux rayons solaires incidents et provoquent un refroidissement, comme celui qui a suivi l'éruption du Pinatubo en 1991 et qui a provoqué une baisse de température moyenne d'un demi-degré pendant deux ans.

\section{Pour en savoir plus}

- J.L. Dufresne et J. Treiner, « L'effet de serre atmosphérique : plus subtil qu'on ne le croit !", La Météorologie, n 72 (février 2011), p. 31.

- C. Jeandel et R. Mosseri, Le Climat à découvert, CNRS Éditions (2011).

- S. Solomon et al. (eds.), Contribution of Working Group I to the Fourth Assessment Report of the Intergovernmental Panel on Climate Change, Cambridge University Press (2007). Le rapport peut être téléchargé sur le site du GIEC www.ipcc.ch/publications_and_data/ar4/wg1/en/ contents.html

- Nous recommandons au lecteur de se reporter, dès sa sortie, au prochain rapport du GIEC, le Fifth Assessment Report of the Intergovernmental Panel on Climate Change, dont la parution est prévue en 2013 et qu'on trouvera alors sur le site du GIEC (http://www.ipcc.ch/).

- Un livre très didactique : M.A. Mélières et C. Maréchal, Climat et société, édité par le CRDP de l'académie de Grenoble (2010). 


\section{Conclusion}

Bien qu'étant présents à des concentrations très faibles dans l'atmosphère, les gaz à effet de serre jouent un rôle fondamental dans l'établissement de la température moyenne à la surface du globe ; un accroissement de leur concentration se traduit automatiquement par une élévation de cette température. Les incertitudes sur les forçages, qui concernent principalement les aérosols et les nuages, ne remettent pas en cause l'impact majeur des gaz à effet de serre sur le réchauffement en cours. La poursuite de l'accumulation de ces derniers dans l'atmosphère conduit à un réchauffement inéluctable de la planète. Il est de ce fait particulièrement inquiétant que les émissions de carbone dans l'atmosphère ne cessent de croître : comme le montre la figure 7 , chaque nouvelle source de $\mathrm{CO}_{2}$ s'est globalement toujours ajoutée aux précédentes, sans jamais s'y substituer. Pour continuer à couvrir nos divers besoins d'énergie, il est urgent de mettre en œuvre des substituts non carbonés mais ayant une souplesse d'emploi comparable aux combustibles fossiles.

(a) Le flux géothermique ne fait que $0,17 \%$ du flux solaire.

(b) L'albédo est la fraction du rayonnement incident qui est réfléchie.

(c) Selon la loi de Stefan-Boltzmann, le flux F émis par un corps à la température $\mathrm{T}$ est : $\mathrm{F}=\sigma \mathrm{T}^{4}$, avec $\sigma=5,6710^{-8} \mathrm{~W} \cdot \mathrm{m}^{-2} \cdot \mathrm{K}^{-4}$.

(d) Pour qu'en l'absence d'effet de serre la température de la Terre soit de $-18^{\circ} \mathrm{C}$, il faudrait qu'il n'y ait pas d'atmosphère mais que l'albédo planétaire, dû pour une part notable aux nuages, reste le même.

(e) Le terme, dû à Arrhenius, est un peu mal choisi : une serre fonctionne par confinement de l'air chaud à l'intérieur de l'enceinte, alors que l'effet de serre est un confinement du rayonnement infrarouge.

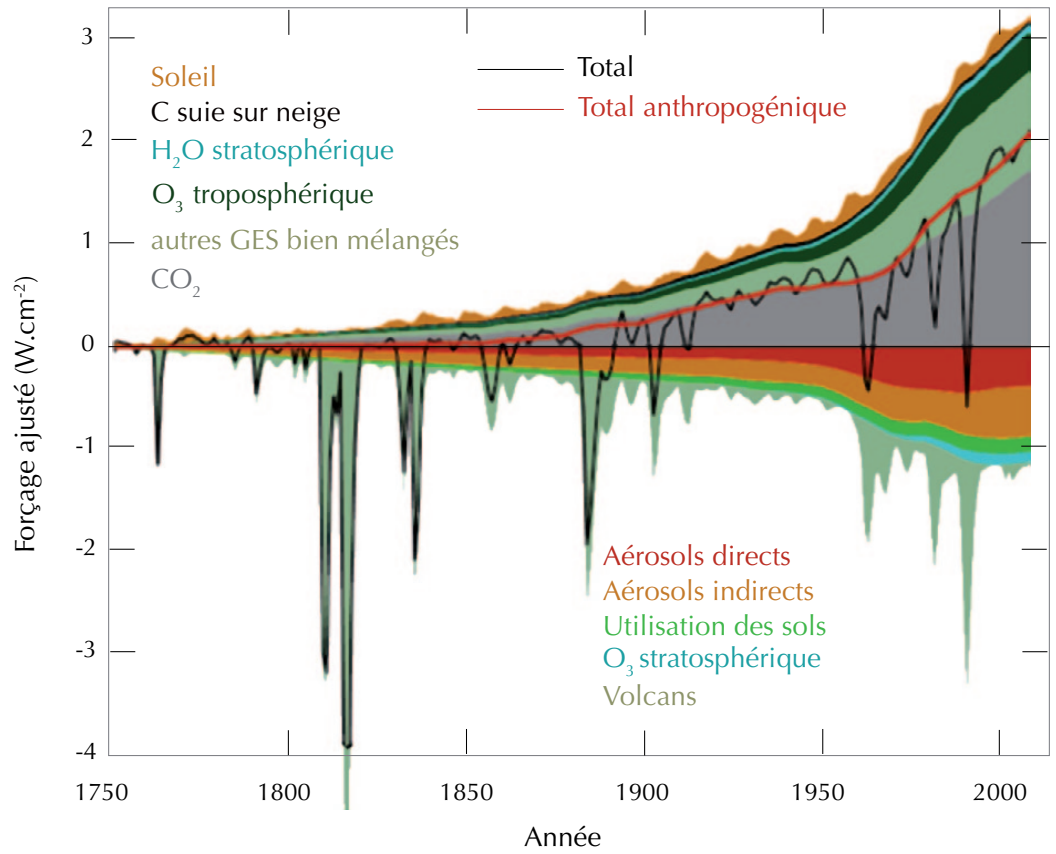

6. Évolution des diverses composantes du forçage depuis 1750.

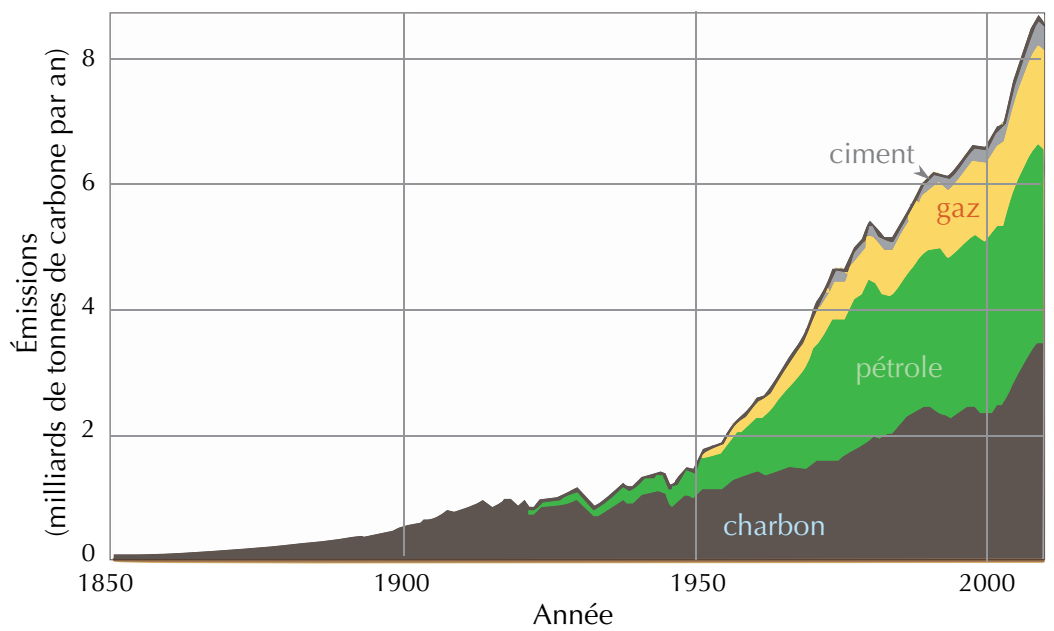

7. Part des différentes émissions mondiales de carbone (hors changement d'utilisation des sols) depuis 1850 .

\section{Aérosols et forçage radiatif}

Tel qu'on l'a défini, le forçage radiatif est un effet instantané. Du fait de ce forçage, la machine climatique va réagir et adapter le climat pour restaurer l'équilibre. Mais ce processus n'est évidemment pas instantané, compte tenu des temps caractéristiques de réponse des différents compartiments de I'environnement terrestre, au cours duquel l'agent forçant peut évoluer et avoir des impacts indirects par action sur d'autres composantes du système climatique. Cela peut se traduire, après un délai plus ou moins long, par d'autres effets radiatifs s'ajoutant ou se retranchant au forçage initial.

Prenons l'exemple des aérosols, ces particules en suspension, dans l'atmosphère. Dès sa formation, l'aérosol exerce une action sur le rayonnement, par diffusion de Mie (diffusion de la lumière par les particules) et, pour certains aérosols par absorption du rayonnement; il s'agit d'un effet radiatif direct. Mais l'absorption de la lumière par un aérosol absorbant va chauffer localement l'atmosphère et en perturber la dynamique. De plus, les particules en suspension sont aussi des noyaux sur lesquels la vapeur d'eau va se condenser pour former les nuages dont l'aérosol va conditionner les propriétés optiques et le temps de résidence dans l'atmosphère (cet effet indirect est la cause majeure de l'effet refroidissant des aérosols).

Pour tenir compte de tous ces effets et de leur impact sur le bilan radiatif, les climatologues ont introduit un nouveau concept : le forçage radiatif ajusté, qui est le bilan des effets radiatifs immédiats et des effets radiatifs différés induits par la présence de l'agent forçant, l'aérosol dans notre exemple. 